Article

\title{
Genotype-Based Gene Expression in Colon Tissue-Prediction Accuracy and Relationship with the Prognosis of Colorectal Cancer Patients
}

\author{
Heike Deutelmoser 1,2 ${ }^{1}$, Justo Lorenzo Bermejo ${ }^{2}$, Axel Benner ${ }^{3}$, Korbinian Weigl ${ }^{4}$, \\ Hanla A. Park ${ }^{5}$, Mariam Haffa 1,6 , Esther Herpel 7,8, Martin Schneider ${ }^{9}$, \\ Cornelia M. Ulrich ${ }^{1,10,11} \mathbb{D}^{D}$, Michael Hoffmeister ${ }^{4}\left(\mathbb{D}\right.$, Jenny Chang-Claude ${ }^{5,12}(\mathbb{D}$, \\ Hermann Brenner 1,4,13 (D) and Dominique Scherer 1,2,*(D)
}

1 Division of Preventive Oncology, German Cancer Research Center (DKFZ) and National Center for Tumor Diseases (NCT), Im Neuenheimer Feld 460, 69120 Heidelberg, Germany; heike.deutelmoser@nct-heidelberg.de (H.D.); mariam.haffa@nct-heidelberg.de (M.H.); neli.ulrich@hci.utah.edu (C.M.U.); h.brenner@dkfz-heidelberg.de (H.B.)

2 Institute of Medical Biometry and Informatics, Medical Faculty, Heidelberg University, Im Neuenheimer Feld 130.3, 69120 Heidelberg, Germany; lorenzo@imbi.uni-heidelberg.de

3 Division of Biostatistics, German Cancer Research Center (DKFZ), Im Neuenheimer Feld 581, 69121 Heidelberg, Germany; benner@dkfz-heidelberg.de

4 Division of Clinical Epidemiology and Aging Research, German Cancer Research Center (DKFZ), Im Neuenheimer Feld 581, 69121 Heidelberg, Germany; k.weigl@dkfz-heidelberg.de (K.W.); m.hoffmeister@dkfz-heidelberg.de (M.H.)

5 Division of Cancer Epidemiology, German Cancer Research Center (DKFZ), Im Neuenheimer Feld 581, 69121 Heidelberg, Germany; h.park@dkfz-heidelberg.de (H.A.P.); j.chang-claude@dkfz-heidelberg.de (J.C.-C.)

6 Division of Translational Functional Cancer Genomics, National Center for Tumor Diseases (NCT) and German Cancer Research Center (DKFZ), Im Neuenheimer Feld 460, 69120 Heidelberg, Germany

7 NCT Tissue Bank, National Center for Tumor Diseases (NCT) and University Hospital Heidelberg, Im Neuenheimer Feld 460, 69120 Heidelberg, Germany; esther.herpel@med.uni-heidelberg.de

8 Institute of Pathology, University Hospital Heidelberg, Im Neuenheimer Feld 224, 69120 Heidelberg, Germany

9 Department of General, Visceral, and Transplantation Surgery, University Hospital Heidelberg, Im Neuenheimer Feld 420, 69120 Heidelberg, Germany; martin.schneider@med.uni-heidelberg.de

10 Huntsman Cancer Institute, 2000 Cir of Hope Dr 1950, Salt Lake City, UT 84112, USA

11 Department of Population Health Sciences, School of Medicine, University of Utah, Salt Lake City, UT 84112, USA

12 Cancer Epidemiology Group, University Cancer Center Hamburg (UCCH), University Medical Center Hamburg-Eppendorf (UKE), Martinstraße 52, 20246 Hamburg, Germany

13 German Cancer Consortium (DKTK), German Cancer Research Center (DKFZ), Im Neuenheimer Feld 280, 69120 Heidelberg, Germany

* Correspondence: scherer@imbi.uni-heidelberg.de; Tel.: +49-6221-56-7302

Received: 30 September 2020; Accepted: 27 October 2020; Published: 31 October 2020

check for updates

\begin{abstract}
Colorectal cancer (CRC) survival has environmental and inherited components. The expression of specific genes can be inferred based on individual genotypes-so called expression quantitative trait loci. In this study, we used the PrediXcan method to predict gene expression in normal colon tissue using individual genotype data from 91 CRC patients and examined the correlation $\rho$ between predicted and measured gene expression levels. Out of 5434 predicted genes, $58 \%$ showed a negative $\rho$ value and only $16 \%$ presented a $\rho$ higher than 0.10 . We subsequently investigated the association between genotype-based gene expression in colon tissue for genes with $\rho>0.10$ and survival of 4436 CRC patients. We identified an inverse association between the predicted expression of $A R I D 3 B$ and CRC-specific survival for patients with a body mass index greater than
\end{abstract}


or equal to $30 \mathrm{~kg} / \mathrm{m}^{2}$ (HR (hazard ratio) $=0.66$ for an expression higher vs. lower than the median, $p=0.005)$. This association was validated using genotype and clinical data from the UK Biobank $(\mathrm{HR}=0.74, p=0.04)$. In addition to the identification of ARID3B expression in normal colon tissue as a candidate prognostic biomarker for obese CRC patients, our study illustrates the challenges of genotype-based prediction of gene expression, and the advantage of reassessing the prediction accuracy in a subset of the study population using measured gene expression data.

Keywords: genotype-based gene expression; PrediXcan; colorectal cancer; survival

\section{Introduction}

Colorectal cancer (CRC) is a leading cause of cancer death worldwide [1,2]. Modifiable factors of colorectal cancer patients' survival include smoking, alcohol consumption, aspirin use, and physical activity, while the effect of obesity is still controversial [3]. In addition, several studies have identified genetic polymorphisms associated with colorectal cancer prognosis [4-8]. The effect of prognostic genetic variants is thought to be, to a large extent, of a regulatory nature, leading to a modulation of the expression of target genes. Single nucleotide polymorphisms (SNP) that modulate gene expression are called expression quantitative trait loci (eQTLs) and may act in cis (modulating the expression of a near-by gene) or in trans (modulating the expression of a distant gene) [9]. Within recent years, immense efforts have been undertaken to map tissue-specific regulatory variants of the human genome $[10,11]$, resulting in a large variety of tools and databases that facilitate the functional characterization of polymorphisms and their proxies identified in genetic association studies [12,13].

The information contained in such databases is the basis of PrediXcan, a method that enables the prediction of tissue-specific gene expression based on individual genotype data [14]. PrediXcan estimates the fraction of genetically determined gene expression levels and performs association analyses between predicted gene expression profiles and a phenotype of interest. This approach potentially accelerates the identification of phenotype-shaping genes. Genome-wide association studies have identified thousands of loci associated with complex traits [15]. The use of PrediXcan is increasingly common in genetic association studies [16-24] and recently contributed to the identification of genes associated with lipid levels and schizophrenia [19,20], cutaneous squamous cell carcinoma [23], lung cancer [24], and colorectal cancer [21]. For example, TRIM4 and PYGL, both related to cellular metabolic programming, were associated with CRC risk [21]. However, studies have also reported that the prediction accuracy may be impaired due to, for example, population stratification $[25,26]$. A limitation of previous studies is that they fully relied on predicted gene expression without consideration of potential differences in prediction accuracy among human populations. Furthermore, to our knowledge, no previous study has used PrediXcan to investigate colorectal cancer prognosis. We thus measured global gene expression profiles in healthy colon mucosa of 91 colorectal cancer patients from the ColoCare-“Darmkrebs: Chancen der Verhuetung durch Screening” (DACHS) study and subsequently inferred gene expression profiles based on individual genotype data using PrediXcan. We calculated the Spearman correlation $\rho$ between the measured and the genetically predicted gene expression levels as a measure of prediction accuracy, and further investigated the association between the genetically-determined gene expression and survival of 4436 colorectal cancer patients for 863 well-predicted genes $(\rho>0.10)$.

\section{Results}

\subsection{Correlation between Measured and Genetically Predicted Gene Expression}

We first examined the correlation between measured and genetically predicted gene expression levels in a subset of 91 colorectal cancer patients, for which genome-wide genotype and gene expression 
data of healthy colorectal mucosa were available (Figure 1). The characteristics of this subset of the total study population are presented in Table S1.

Out of 159,506 SNPs that PrediXcan uses to predict gene expression in colon transverse tissue, 158,115 SNPs with a genotype imputation score higher than 0.99 were available in our study of 91 participants and were used for the prediction of gene expression in normal colon tissue. This translated into the estimation of gene expression levels of 6304 genes, while measured gene expression data in normal tissue was available for 5434 genes. Figure 2a shows the mean measured (x-axis) versus the mean predicted (y-axis) expression values for the 5434 investigated genes.

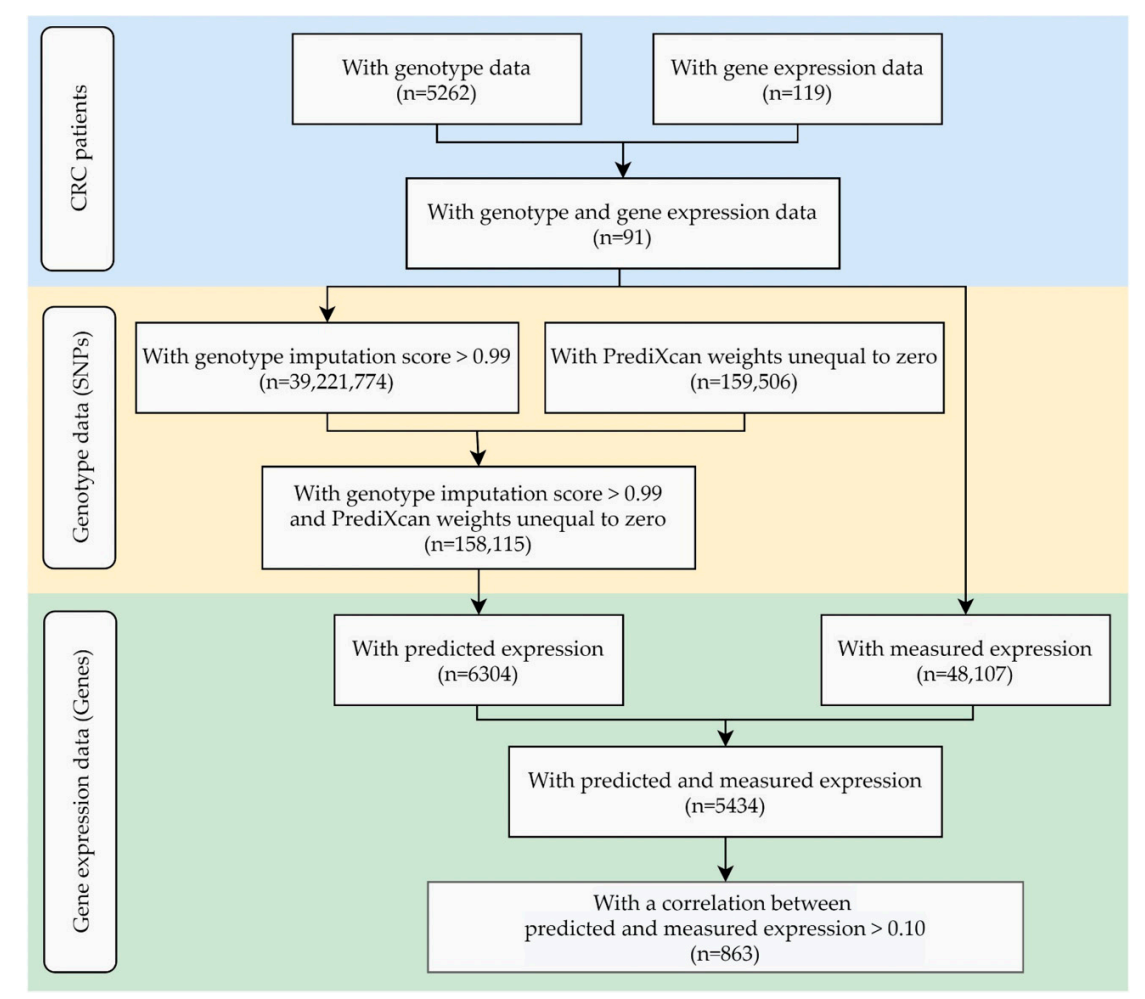

Figure 1. Data overview for the examination of the correlation $\rho$ between predicted and measured gene expression. Abbreviations: CRC: colorectal cancer; SNPs: single nucleotide polymorphisms.

The correlation between measured and genetically predicted gene expression among the 91 investigated individuals was negative for $58 \%$ of the genes (displayed in red) and between 0 and 0.10 for $26 \%$ of the genes (displayed in black), and only $16 \%$ (863 genes) presented a correlation higher than 0.10 (displayed in green, listed in Table S2). 


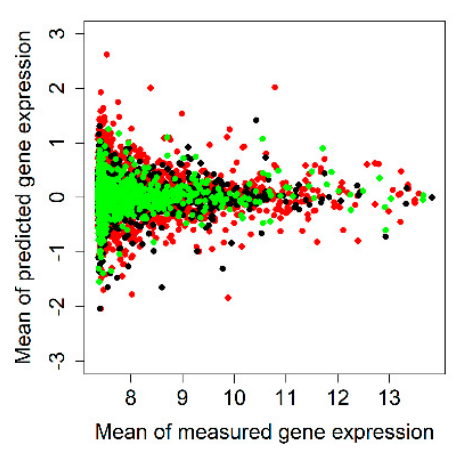

(a)

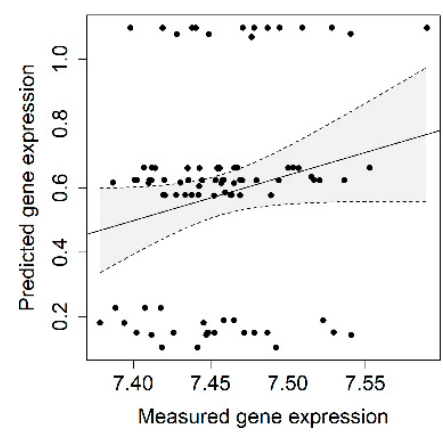

(b)

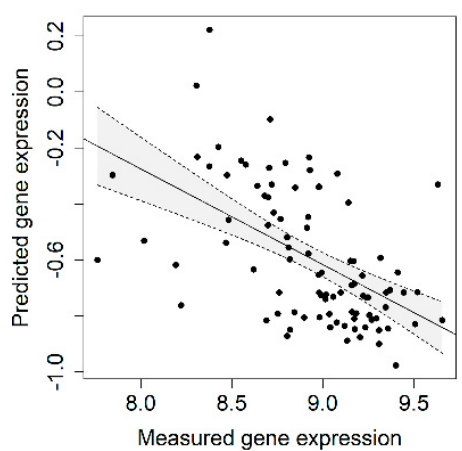

(c)

Figure 2. (a) Scatter plot of the mean measured versus the mean predicted expression values for 5434 genes with estimated Spearman correlation coefficients $Q<0$ in red, $0 \leq \mathrm{Q} \leq 0.1$ in black, and $Q>0.1$ in green; (b,c) measured versus predicted expression values for the genes TRIM4 (b), and PYGL (c). The linear regression lines are also shown with their corresponding $95 \%$ confidence bands.

PrediXcan has been previously applied to identify novel colorectal cancer risk loci [21,22]. Since the preceding studies included a part of the study population presented here, we investigated the accuracy of the predicted gene expression levels for the previously identified genes. These previous studies reported that the expression of TRIM4 and PYGL in colon transverse tissue was associated with colorectal cancer risk [21], while PTPN2 expression in colon transverse tissue modified the association of diabetes with colorectal cancer risk [22]. The expression of PTPN2 was not measured in our study of 91 participants and could thus not be further investigated. However, we examined the correlation between measured and predicted gene expression for TRIM4 and PYGL (Figure 2b-c) and observed a correlation of $\mathrm{Q}=0.19$ for TRIM4 and a negative correlation of $\mathrm{Q}=-0.56$ for PYGL. The SNPs as well as the corresponding regression coefficients used to predict the gene expression of TRIM4 and PYGL are provided in Table S3.

\subsection{Association of Genetically Predicted Gene Expression and Colorectal Cancer Patients' Survival}

We then investigated the association between the genotype-based gene expression for genes with a good prediction accuracy (863 genes with a correlation higher than $\varrho=0.10$ ) and survival of 4436 colorectal cancer patients. Characteristics of the study population for the investigated endpoints overall survival (OS) and disease-specific survival (DSS) are presented in Table 1.

During a median follow up of 6.94 years, 1790 patients died and 1053 died of colorectal cancer. Patients who died from any cause (overall survival) were older, diagnosed at higher stage of the disease, were more likely to be also affected with diabetes, and were less likely to consume alcohol or to be overweight or obese.

The genetically predicted expression of 36 genes was associated with overall survival and of 48 genes with disease-specific survival (Table S4). After adjustment for multiple testing, the smallest probability value was found for the association between the genetically predicted expression of $M A P 1 B$ and disease-specific survival (raw $p=0.0002$, multiplicity-adjusted $p=0.15$ ).

We further performed stratified analyses. Since body mass index (BMI) was the only variable associated with overall and disease-specific survival, which was also available in the UK Biobank, we classified colorectal cancer patients into four BMI groups: BMI $<18.5 \mathrm{~kg} / \mathrm{m}^{2}=$ underweight, BMI 18.5-24.9 kg/m ${ }^{2}=$ normal weight, BMI $25-29.9 \mathrm{~kg} / \mathrm{m}^{2}=$ overweight, and BMI $\geq 30 \mathrm{~kg} / \mathrm{m}^{2}=$ obese, and investigated the association between genetically determined gene expression and survival of colorectal cancer patients in each BMI category. 
Table 1. Characteristics of the study population.

\begin{tabular}{|c|c|c|c|c|c|c|c|c|}
\hline \multirow[b]{2}{*}{ Variable } & \multirow[b]{2}{*}{ Level } & \multirow[b]{2}{*}{ Patients } & \multicolumn{3}{|c|}{ Overall Survival } & \multicolumn{3}{|c|}{ CRC-Specific Survival } \\
\hline & & & Deaths & $\mathrm{HR}^{1}(95 \% \mathrm{CI})$ & $p$ & Deaths & $\mathrm{HR}^{1}(95 \% \mathrm{CI})$ & $p$ \\
\hline Age at & $<60$ & 946 & 257 & Ref. & $<2 \times 10^{-16}$ & 209 & Ref. & 0.0003 \\
\hline \multirow{3}{*}{$\begin{array}{l}\text { Diagnosis } \\
\text { (years) }\end{array}$} & $60-69$ & 1339 & 466 & $1.25(1.07-1.46)$ & & 314 & $1.06(0.89-1.27)$ & \\
\hline & $70-79$ & 1489 & 638 & $1.69(1.47-1.96)$ & & 357 & $1.12(0.96-1.36)$ & \\
\hline & $>80$ & 662 & 429 & $3.20(2.74-3.74)$ & & 173 & $1.47(1.20-1.80)$ & \\
\hline \multirow[t]{2}{*}{ Gender } & Male & 2685 & 1111 & Ref. & 0.16 & 626 & Ref. & 0.47 \\
\hline & Female & 1751 & 679 & $1.07(0.97-1.18)$ & & 427 & $0.96(0.85-1.08)$ & \\
\hline \multirow[t]{4}{*}{ CRC stage } & I & 1024 & 235 & Ref. & $<2 \times 10^{-16}$ & 40 & Ref. & $\begin{array}{l}<2 \times \\
10^{-16}\end{array}$ \\
\hline & II & 1345 & 434 & $1.49(1.27-1.74)$ & & 148 & $2.96(2.09-4.20)$ & \\
\hline & III & 1426 & 575 & $2.05(1.76-2.39)$ & & 354 & $7.29(5.25-10.10)$ & \\
\hline & IV & 641 & 546 & 10.21 & & 511 & 48.91 & \\
\hline \multirow[t]{2}{*}{ Tumor site } & Colon & 2665 & 1069 & $\begin{array}{c}\text { (8.73-11.94) } \\
\text { Ref. }\end{array}$ & 0.61 & 598 & $\begin{array}{c}\text { (35.37-67.64) } \\
\text { Ref. }\end{array}$ & 0.03 \\
\hline & Rectum & 1771 & 721 & $1.03(0.93-1.13)$ & & 455 & $1.15(1.02-1.30)$ & \\
\hline \multirow{4}{*}{$\begin{array}{c}\text { Body mass } \\
\text { index } \\
\left(\mathrm{kg} / \mathrm{m}^{2}\right)\end{array}$} & $<18.5$ & 121 & 71 & $1.74(1.37-2.23)$ & 0.0006 & 41 & $1.62(1.17-2.23)$ & 0.01 \\
\hline & $18.5-24.9$ & 1592 & 675 & Ref. & & 396 & Ref. & \\
\hline & $25-29.9$ & 1871 & 726 & $0.87(0.78-0.96)$ & & 432 & $0.88(0.77-1.01)$ & \\
\hline & $\geq 30$ & 852 & 318 & $0.83(0.73-0.95)$ & & 184 & $0.83(0.70-0.99)$ & \\
\hline \multirow[t]{2}{*}{ Diabetes } & No & 3594 & 1360 & Ref. & $<8 \times 10^{-13}$ & 844 & Ref. & 0.09 \\
\hline & Yes & 811 & 412 & $1.50(1.34-1.67)$ & & 200 & $1.14(0.98-1.33)$ & \\
\hline Regular & No & 3272 & 1298 & Ref. & 0.05 & 793 & Ref. & 0.31 \\
\hline NSAID use & Yes & 1105 & 469 & $1.11(1.00-1.24)$ & & 245 & $0.93(0.80-1.07)$ & \\
\hline \multirow{3}{*}{ Smoking } & Never & 1921 & 840 & Ref. & 0.04 & 515 & Ref. & 0.07 \\
\hline & Former & 1769 & 684 & $0.90(0.81-1.00)$ & & 367 & $0.79(0.69-0.90)$ & \\
\hline & Current & 672 & 263 & $0.89(0.78-1.03)$ & & 169 & $0.94(0.79-1.12)$ & \\
\hline \multirow{5}{*}{$\begin{array}{l}\text { Alcohol } \\
\text { intake } \\
\text { (g/day) }\end{array}$} & $\begin{array}{c}\text { No } \\
\text { intake }\end{array}$ & 1320 & 606 & Ref. & 0.0003 & 339 & Ref. & 0.01 \\
\hline & $0.1-5.6$ & 856 & 318 & $0.75(0.66-0.86)$ & & 202 & $0.87(0.73-1.03)$ & \\
\hline & $5.7-13.2$ & 770 & 280 & $0.72(0.63-0.83)$ & & 175 & $1.22(0.68-0.98)$ & \\
\hline & $13.3-28.5$ & 775 & 301 & $0.78(0.68-0.90)$ & & 177 & $1.20(0.70-1.00)$ & \\
\hline & $\geq 28.6$ & 669 & 269 & $0.79(0.69-0.92)$ & & 148 & $1.24(0.66-0.97)$ & \\
\hline
\end{tabular}

${ }^{1}$ Hazard ratios adjusted for age, sex, CRC stage, and tumor site. Abbreviations: HR: hazard ratio; CI: confidence interval; CRC: colorectal cancer; Ref.: reference category; NSAID: non-steroidal anti-inflammatory drug.

The expression of more than thirty genes was associated with overall or disease-specific survival of colorectal cancer patients in each BMI group (Table S4). After adjustment for multiple testing, the genetically predicted expression of $A R I D 3 B$ showed the strongest association with disease-specific survival (raw $p=0.00008$, multiplicity-adjusted $p=0.07$ ).

Figure 3 shows the volcano plots of the survival analyses of 852 obese CRC patients in the DACHS study (Figure 3a: overall survival, Figure 3b: disease-specific survival) with the blue dots indicating the results for the gene ARID3B. The correlation between the measured and the genotype-based ARID3B expression was $\rho=0.11$.

We were further able to validate this association in an independent dataset of 1115 colorectal cancer patients with a BMI higher than or equal to $30 \mathrm{~kg} / \mathrm{m}^{2}$ in the UK Biobank $(p=0.02)$. The characteristics of the total study population of the validation dataset are presented in Table S5 (median follow up of 7.02 years; 1035 deaths, 669 deaths due to CRC).

Figure $3 c$, d depicts the disease-specific survival of obese colorectal cancer patients according to predicted expression of $A R I D 3 B$ in the discovery (Figure $3 c$, HR (hazard ratio) $=0.66 ; p=0.005$ for an expression higher vs. lower than the median) and validation datasets (Figure $3 \mathrm{~d}, \mathrm{HR}=0.74 ; p=0.04$ ). In both the identification and the validation datasets, only obese CRC patients showed an association between genetically predicted $A R I D 3 B$ expression and disease-specific survival (Figure S1). 


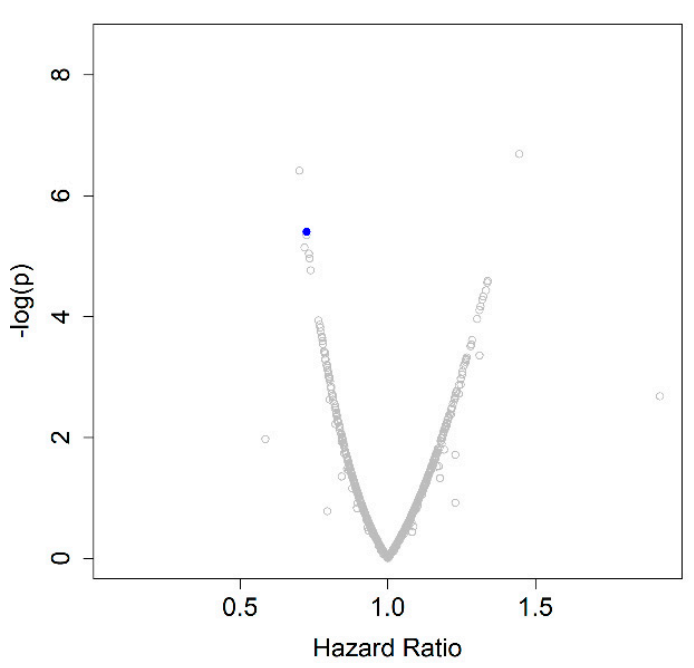

(a)

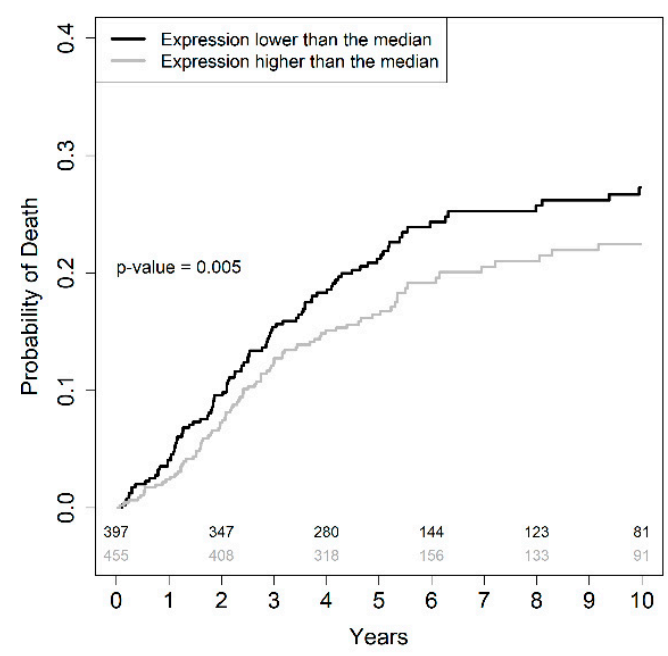

(c)

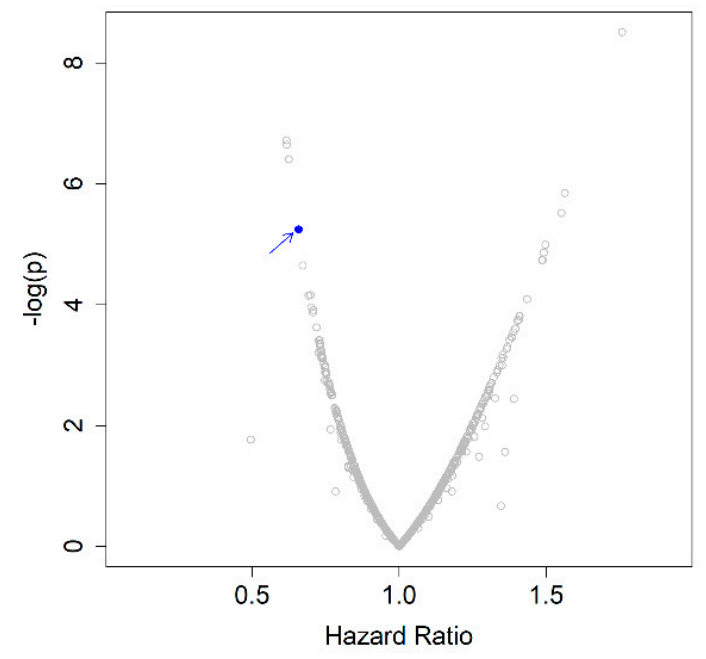

(b)

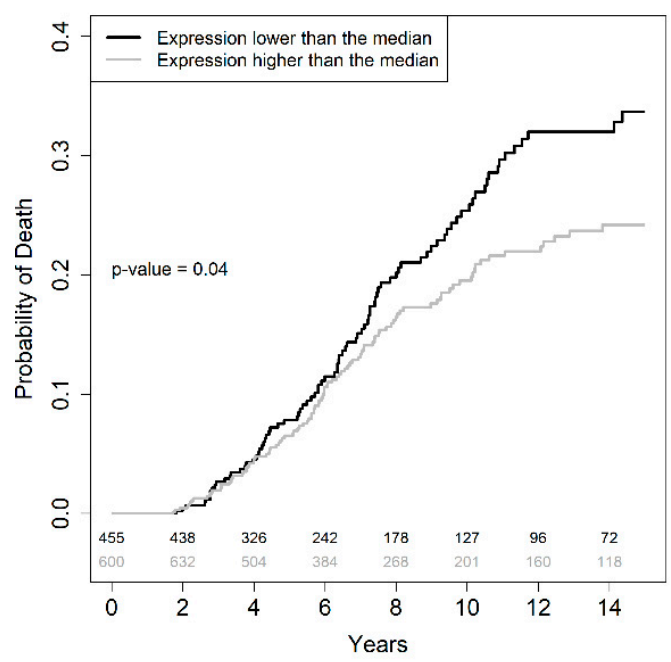

(d)

Figure 3. (a,b) Volcano plots showing the results from survival analyses of 852 CRC patients with a $\mathrm{BMI} \geq 30$, and 863 genes with a correlation between predicted and measured expression values higher than 0.10. The blue dots indicate results for the gene ARID3B. (a) Overall survival; (b) CRC-specific survival; (c,d) cumulative probability of death due to CRC for patients with a low (lower than the median) and high (higher than the median) ARID3B expression based on individual genotypes for patients with $B M I \geq 30$. The number of CRC patients at risk is shown in the lower part of each panel; (c) Aalen-Johansen probability curves in the identification cohort ("Darmkrebs: Chancen der Verhuetung durch Screening" (DACHS) study); (d) Aalen-Johansen probability curves in the validation cohort (UK Biobank). Abbreviations: CRC: colorectal cancer.

\section{Discussion}

In the present study, we used PrediXcan to infer gene expression in normal colon tissue from 91 colorectal cancer patients and examined the prediction accuracy by comparing the predicted and the measured gene expression levels. We then investigated the association between gene expression for well-predicted genes $(\rho>0.10)$ and survival of 4436 colorectal cancer patients from the DACHS study. We observed that out of 5434 genes, for which measured as well as predicted gene expression data were available, $58 \%$ showed a negative correlation, while only $16 \%$ presented a correlation higher 
than 0.10 and were thus taken forward to perform association analyses with the survival of colorectal cancer patients.

The PrediXcan prediction model uses a median number of 23 SNPs to predict gene expression (minimum $=1$, maximum $=259$ SNPs). The expression of PYGL was predicted based on seven SNPs, which should suffice to accurately predict gene expression. The negative correlation between measured and predicted expression of PYGL $(\rho=-0.58)$ in our study is unlikely the result of an insufficient number of eQTLs as previous studies reported that genetically regulated gene expression seems to be associated with a small number of variants rather than with multiple eQTLs [27,28]. Nevertheless, the expression of better predicted gene TRIM4 $(\rho=0.19)$ was based on 26 genetic variants.

Negative correlations between measured and genetically predicted gene expression using PrediXcan have been reported in previous studies $[25,26]$ and could also result from weak associations between SNPs and the expression levels of the target gene. Finally, PrediXcan prediction models were trained based on local SNPs (cis-eQTLs) within one megabase (MB) of the start or the end of the gene, and the inclusion of potential trans-eQTLs could improve the prediction of gene expression.

Recent studies reported that gene expression prediction accuracy varies between populations $[25,26]$. The reference datasets, which were used to train the PrediXcan prediction models, are the Depression Genes and Networks (DGN) study and the Genotype-Tissue Expression (GTEx) project. The majority of the subjects in these reference datasets are of European descent [10]. Thus, it is not surprising that PrediXcan predicts gene expression in individuals of European descent more accurately than individuals of African descent [26]. However, differences in prediction accuracy were also reported among closely related European populations [26]. Our data corroborates this observation in showing that the correlation between measured and genetically predicted gene expression within Europeans strongly varies for some genes. PYGL, which was previously associated with colorectal cancer risk, showed a correlation of 0.51 between measured and predicted gene expression based on data from GTEx [21]. In contrast, in our data we observed a strong negative correlation between measured and predicted gene expression for PYGL $(\rho=-0.58)$, implying that subpopulation differences may also be present in Europeans and need to be considered in genetic prediction tools [26,29]. Alternatively, we propose to measure gene expression in a subset of the total study population to assure that the correlation between predicted and measured gene expression levels of the investigated genes is greater than a preset threshold for the correlation (here 0.10 ) and to filter for well-predicted genes.

After we filtered for well-predicted genes, we identified one gene that was associated with survival of colorectal cancer patients and was subsequently validated in an independent dataset using genotype and clinical data from the UK Biobank. An increased expression of ARID3B was associated with a better disease-specific survival of obese colorectal cancer patients. ARID3B (AT-Rich Interaction Domain 3B) encodes for a DNA binding protein and has been described as contributor to tumor initiation and progression in cancerous diseases [30]. A recent study has described the role of ARID3B in colorectal tumor growth [31]. ARID3B has been further described as an oncoprotein and is involved in the progression of malignant neuroblastoma, ovarian cancer, and breast cancer [32-35]. However, to our knowledge no association of $A R I D 3 B$ with overweight or obesity has been reported, and it is unclear why this association was restricted to obese patients and not observed in other BMI-groups.

The present study is based on 4434 colorectal cancer cases with detailed clinical, demographic, and genome-wide genotype data, as well as with global gene expression data for a subset of 91 patients. The availability of paired genotype and gene expression data for a subset of patients enabled us to investigate the correlation between measured and genetically predicted gene expression, which is a major strength of this study. We were thus able to filter for well-predicted genes within our population to subsequently perform association analyses. Furthermore, we had access to an independent dataset from the UK Biobank, in which we validated the association between ARID3B expression and disease-specific survival of obese colorectal cancer patients. Although our sample size was fairly large, some of the subgroup analyses were based on small strata and this hampered stratified correlation analyses between measured and predicted gene expression. Furthermore, we did not have access to measured 
gene expression data from the validation dataset to test for the accuracy of the predicted expression of ARID3B.

In conclusion, this study illustrates the challenges of gene expression prediction in normal tissue based on individual genotype data and underlines the importance of assessing prediction accuracy through measuring gene expression in a subset of the investigated study population. Finally, we identified $A R I D 3 B$ as a potential survival-modifier in obese colorectal cancer patients.

\section{Materials and Methods}

\subsection{Study Population}

The study population included patients with CRC who participated in a long-term follow-up study of patients of the German population-based case-control DACHS study ("Darmkrebs: Chancen der Verhuetung durch Screening") [36,37]. CRC patients with a primary, confirmed diagnosis of CRC had been recruited from hospitals of the Rhein-Neckar-Odenwald region since January 2003. Included were patients aged 30 years or older, German speaking, resident in the study region, and mentally and physically able to complete an in-person interview. Baseline standardized questionnaires contained demographic information and information on established or suggested CRC risk factors, as well as possible prognostic factors. Follow-up information on overall and disease-specific survival was collected at three, five, and ten years after diagnosis. Causes of death were verified by death certificates and coded based on ICD-10 classifications. Information on recurrences were collected from general practitioners and specialists as applicable. In addition, clinical data was extracted from patient records. Population controls were randomly selected from lists of residents of the population registries of the cities and counties. The study was approved by the ethics committee (approved on 6 December 2001, project identification code 310/2001) of the University of Heidelberg and State Medical Boards of Baden-Wuerttemberg and was conducted in agreement with the Helsinki Declaration. Written informed consent was provided by all participants at baseline and during follow-up.

\subsection{Genotyping}

5262 DACHS samples were genotyped using the whole-genome Illumina CytoSNP assay (Illumina, San Diego, CA, USA) for patients recruited 2003-2007, the Illumina HumanOmniExpress BeadChip Kit for patients recruited 2008-2014, and the Illumina Infinium OncoArray-500K BeadChip for those recruited 2015 or the Infinium Global Screening Array for patients recruited 2016-2017 as described previously [38]. Missing genotypes were imputed using the Haplotype Reference Consortium as reference panel 1 (HRC r1.1 2016).

\subsection{Gene Expression Measurement}

Gene expression profiles of healthy colorectal mucosa tissues from 91 participants of a subsample of the DACHS study (ColoCare-DACHS study) were measured using Illumina HumanHT-12 Expression BeadChips according to the manufacturer's instructions as described previously $[39,40]$. Raw gene expression data was processed prior to statistical analyses. Missing expression values were imputed using the nearest neighbor averaging method as implemented in the $\mathrm{R}$ package impute. Expression data were adjusted for batch effects using the R package "sva" and subsequently transformed using the variance stabilizing transformation method and normalized using the robust spline normalization method of the R package "lumi".

\subsection{Gene Expression Prediction}

Out of the 4465 CRC patients of the DACHS study with available genome-wide genotype and follow-up data, we used 4436 CRC patients with available information on age, gender, stage at diagnosis and tumor site, and a minimum follow-up of 30 days. 
DACHS genotype data was combined and quality controlled using the info score of the program qctool (https://www.well.ox.ac.uk/ gav/qctool_v2). PrediXcan transcriptome prediction models were downloaded from the publicly available PredictDB repository (www.predictdb.org). Gene expression profiles in colon normal tissue were estimated based on genome-wide genotype data of DACHS patients using the software PrediXcan (https://github.com/hakyimlab/PrediXcan) [14]. PrediXcan provides prediction models trained by elastic net models and using reference datasets from the Genotype-Tissue Expression Project (GTEx), where the majority of subjects are of European descent. The reference dataset for normal colon tissue contained 169 colon transverse samples resulting in 159,506 variants with a prediction weight unequal to zero and used for the gene expression prediction of 6304 colon genes in normal tissue [10].

As the proportion of genetically determined gene expression levels may differ by population, we further tested the accuracy of predicted gene expression levels in the DACHS study. Observed gene expression profiles in healthy colorectal mucosa tissues of 91 CRC patients from the ColoCare-DACHS study were correlated with the predicted gene expression profiles.

Genes with available measured gene expression and with a median absolute deviation (MAD) of measured and predicted gene expression greater than 0 were included. For each gene, a quality metric $\rho$ was computed as the Spearman correlation between the observed and predicted expression. Association tests were restricted to genes with a predictive $\rho \geq 0.10$, i.e., $\geq 10 \%$ correlation between predicted and observed expression (cf. [21,22]).

\subsection{Statistical Analysis}

We tested the association between genetically determined expression levels in healthy colon tissue and the survival of 4436 CRC patients from the DACHS study in 863 genes with a predictive $\rho \geq 0.10$. Cox proportional hazards regression was performed to test the predicted gene expression associations with overall survival and disease-specific survival. Survival time was calculated from date of diagnosis until date of death by any cause for overall survival and from date of diagnosis until date of death by CRC for disease-specific survival. Age, sex, stage at diagnosis, and cancer site were included in the model as relevant prognostic factors. Individuals with missing entries were excluded. The proportional hazards assumption was tested according to Grambsch and Therneau for a set of variables including age ( $<60$ years, 60-69 years, 70-79 years, $\geq 80$ years), sex, stage at diagnosis (I, II, III, IV), cancer site (colon, rectum), chemotherapy (yes, no), diabetes (yes, no), BMI (<18.5, 18.5-24.9, $25-29.9, \geq 30 \mathrm{~kg} / \mathrm{m}^{2}$ ), regular use of non-steroidal anti-inflammatory drugs (NSAIDs) more than twice per week for at least one year (yes, no), regular smoking (never, former, and current) and alcohol intake (0, 0-5.6, 5.7-13.2, 13.3-28.5, and $\geq 28.6 \mathrm{~g} /$ day). Survival analyses were evaluated using as predictor variables the predicted gene expression levels as continuous variables and subsequently the predicted gene expression levels dichotomized at the median level for Cox proportional hazard models to estimate hazard ratios (HR) for overall survival and disease-specific survival, and their $95 \%$ confidence intervals (CIs). The median follow-up time and the cumulative probability of death were calculated using the Aalen-Johansen estimator. Bonferroni-Holm correction method was used to adjust for multiple testing.

The statistical analysis was carried out using SAS version 9.3 (SAS Institute, Cary, NC, USA) and $R$ version 3.1.0 (www.r-project.org).

\subsection{Validation Set}

Validation of associations was performed using the UK Biobank resource. UK Biobank recruited 500,000 people from the UK aged between 40 and 69 years in 2006-2010 [41]. Genotype calling was performed by Affymetrix on the UK BiLEVE Axiom array and the UK Biobank Axiom array (Affymetrix, Santa Clara, CA, USA). Genotypes were imputed using the Haplotype Reference Consortium and UK10K haplotype resources [42]. 
The validation set included 4241 CRC patients from the UK Biobank resource (Table S5). Statistical analyses from our respective findings were performed as described above. Analyses regarding the registered endpoints overall and disease-specific survival were adjusted for the available variables age and sex. Subgroup analyses for the variable BMI were performed.

Supplementary Materials: Supplementary materials can be found at http://www.mdpi.com/1422-0067/21/21/8150/ s1. Table S1: Characteristics of the 91 colorectal cancer patients with measured gene expression data in normal tissue, Table S2: List of 863 well-predicted genes $(\rho>0.10)$, Table S3: SNPs and their corresponding weights used for the prediction of the genes TRIM4 and PYGL (extracted from the PrediXcan transverse colon prediction model), Table S4a: $p$-values from the Cox regression for the association between the continuous predicted gene expression and survival for overall survival (OS), Table S4b: $p$-values from the Cox regression for the association between the continuous predicted gene expression and disease-specific survival (DSS), Table S5: Characteristics of the 4241 colorectal cancer patients from the validation set, Figure S1: Probability of death due to CRC for patients with a low (lower than the median) and high (higher than the median) ARID3B expression based on individual genotypes. The number of CRC patients at risk is shown in the lower part of each panel; $(\mathrm{a}, \mathrm{b})$ Aalen-Johansen curves in the identification cohort in patients with (a) a BMI between 18.5-24.9 (b) a BMI between 25-29.9; (c,d) Aalen-Johansen curves in the validation cohort in patients with (c) a BMI between 18.5-24.9 (d) a BMI between 25-29.9. Aalen-Johansen curves of patients with a BMI $<18.5$ are not shown due to low sample size.

Author Contributions: Conceptualization, J.L.B., H.B., and D.S.; Data curation, K.W., H.A.P., M.H. (Mariam Haffa), and M.H. (Michael Hoffmeister); Formal analysis, H.D.; Funding acquisition, H.B. and D.S.; Investigation, H.D., J.L.B., and D.S.; Methodology, H.D., J.L.B., A.B., and D.S.; Project administration, M.H. (Michael Hoffmeister), J.C.-C., and H.B.; Resources, E.H., M.S., C.M.U., M.H. (Michael Hoffmeister), J.C.-C., and H.B.; Supervision, J.L.B., H.B., and D.S.; Validation, H.D. and D.S.; Visualization, H.D., J.L.B., and D.S.; Writing-original draft, H.D. and D.S.; Writing-review and editing, H.D., J.L.B., A.B., K.W., H.A.P., M.H. (Mariam Haffa), E.H., M.S., C.M.U., M.H. (Michael Hoffmeister), J.C.-C., H.B., and D.S. All authors have read and agreed to the published version of the manuscript.

Funding: This work was supported by grants from the Federal Ministry of Education and Research Germany (BMBF) (grant numbers 01ER1505B to H.B., 01KT1510 to D.S.). C.M.U. was supported by the Huntsman Cancer Foundation and by National Institutes of Health Grants U01 CA206110, R01 CA189184, R01 CA207371, and P30 CA042014. The Heidelberg ColoCare Study was supported by the National Institutes of Health Grant R01 CA189184, the Matthias Lackas Foundation, the Claussen Simon Stiftung, the German Consortium of Translational Cancer Research (DKTK), institutional funding from the German Cancer Research Center (DKFZ) Heidelberg, and the Helmholtz Association (portfolio theme "Metabolic Dysfunction"). Part of this research was conducted using the UK Biobank resource under the Application number 58030.

Acknowledgments: We thank all participants of the DACHS study, the interviewers, as well as the physicians and staff of the following hospitals and cooperating institutions: Chirurgische Universitätsklinik Heidelberg, Klinik am Gesundbrunnen Heilbronn, St. Vincentiuskrankenhaus Speyer, St. Josefskrankenhaus Heidelberg, Chirurgische Universitätsklinik Mannheim, Diakonissenkrankenhaus Speyer, Krankenhaus Salem Heidelberg, Kreiskrankenhaus Schwetzingen, St. Marien und St. Annastiftkrankenhaus Ludwigshafen, Klinikum Ludwigshafen, Stadtklinik Frankenthal, Diakoniekrankenhaus Mannheim, Kreiskrankenhaus Sinsheim, Klinikum am Plattenwald Bad Friedrichshall, Kreiskrankenhaus Weinheim, Kreiskrankenhaus Eberbach, Kreiskrankenhaus Buchen, Kreiskrankenhaus Mosbach, Enddarmzentrum Mannheim, Kreiskrankenhaus Brackenheim, and the Cancer Registry of Rhineland-Palatinate. We thank the microarray unit of the Genomics and Proteomics Core Facility of the German Cancer Research Center for genotyping, particularly Matthias Schick, and also thank Muhabbet Celik, Ursula Eilber, Sabine Behrens, and Ute Handte-Daub for excellent technical assistance. The authors thank the National Center for Tumor Diseases Tissue Biobank for sample handling and storage, and the Microarray Unit of the DKFZ Genomics and Proteomics Core Facility for providing the Illumina Whole-Genome Expression BeadChips and related services.

Conflicts of Interest: The authors declare no conflict of interest. The funders had no role in the design of the study; in the collection, analyses, or interpretation of data; in the writing of the manuscript, or in the decision to publish the results.

\section{References}

1. Bray, F.; Ferlay, J.; Soerjomataram, I.; Siegel, R.L.; Torre, L.A.; Jemal, A. Global cancer statistics 2018: GLOBOCAN estimates of incidence and mortality worldwide for 36 cancers in 185 countries. CA Cancer J. Clin. 2018, 68, 394-424. [CrossRef] [PubMed]

2. Ferlay, J.; Soerjomataram, I.; Dikshit, R.; Eser, S.; Mathers, C.; Rebelo, M.; Parkin, D.M.; Forman, D.; Bray, F. Cancer incidence and mortality worldwide: Sources, methods and major patterns in GLOBOCAN 2012. Int. J. Cancer 2015, 136, E359-E386. [CrossRef] [PubMed] 
3. Brenner, H.; Chen, C. The colorectal cancer epidemic: Challenges and opportunities for primary, secondary and tertiary prevention. Br. J. Cancer 2018, 119, 785-792. [CrossRef] [PubMed]

4. Song, N.; Kim, K.; Shin, A.; Park, J.W.; Chang, H.J.; Shi, J.; Cai, Q.; Kim, D.Y.; Zheng, W.; Oh, J.H. Colorectal cancer susceptibility loci and influence on survival. Genes. Chromosomes Cancer 2018, 57, 630-637. [CrossRef] [PubMed]

5. Dimberg, J.; Shamoun, L.; Landerholm, K.; Andersson, R.E.; Kolodziej, B.; Wågsäter, D. Genetic Variants of the IL2 Gene Related to Risk and Survival in Patients with Colorectal Cancer. Anticancer Res. 2019, 39, 4933-4940. [CrossRef] [PubMed]

6. Summers, M.G.; Maughan, T.S.; Kaplan, R.; Law, P.J.; Houlston, R.S.; Escott-Price, V.; Cheadle, J.P. Comprehensive analysis of colorectal cancer-risk loci and survival outcome: A prognostic role for CDH1 variants. Eur. J. Cancer 2020, 124, 56-63. [CrossRef] [PubMed]

7. Jiraskova, K.; Hughes, D.J.; Brezina, S.; Gumpenberger, T.; Veskrnova, V.; Buchler, T.; Schneiderova, M.; Levy, M.; Liska, V.; Vodenkova, S.; et al. Functional Polymorphisms in DNA Repair Genes Are Associated with Sporadic Colorectal Cancer Susceptibility and Clinical Outcome. Int. J. Mol. Sci. 2018, 20, 97. [CrossRef] [PubMed]

8. Scherer, D.; Deutelmoser, H.; Balavarca, Y.; Toth, R.; Habermann, N.; Buck, K.; Kap, E.J.; Botma, A.; Seibold, P.; Jansen, L.; et al. Polymorphisms in the Angiogenesis-Related Genes EFNB2, MMP2 and JAG1 Are Associated with Survival of Colorectal Cancer Patients. Int. J. Mol. Sci. 2020, 21, 5395. [CrossRef]

9. Westra, H.J.; Franke, L. From genome to function by studying eQTLs. Biochim. Biophys. Acta 2014, 1842, 1896-1902. [CrossRef]

10. Consortium, G.T. The Genotype-Tissue Expression (GTEx) project. Nat. Genet. 2013, 45, 580-585. [CrossRef]

11. Consortium, E.P. An integrated encyclopedia of DNA elements in the human genome. Nature 2012, 489, 57-74. [CrossRef] [PubMed]

12. Boyle, A.P.; Hong, E.L.; Hariharan, M.; Cheng, Y.; Schaub, M.A.; Kasowski, M.; Karczewski, K.J.; Park, J.; Hitz, B.C.; Weng, S.; et al. Annotation of functional variation in personal genomes using RegulomeDB. Genome Res. 2012, 22, 1790-1797. [CrossRef] [PubMed]

13. Variant Effect Predictor. Available online: http://www.ensembl.org/Homo_sapiens/Tools/VEP?db=core (accessed on 14 September 2020).

14. Gamazon, E.R.; Wheeler, H.E.; Shah, K.P.; Mozaffari, S.V.; Aquino-Michaels, K.; Carroll, R.J.; Eyler, A.E.; Denny, J.C.; Consortium, G.T.; Nicolae, D.L.; et al. A gene-based association method for mapping traits using reference transcriptome data. Nat. Genet. 2015, 47, 1091. Available online: https://www.nature.com/articles/ ng.3367\#supplementary-information (accessed on 23 September 2019). [CrossRef]

15. Buniello, A.; MacArthur, J.A.L.; Cerezo, M.; Harris, L.W.; Hayhurst, J.; Malangone, C.; McMahon, A.; Morales, J.; Mountjoy, E.; Sollis, E.; et al. The NHGRI-EBI GWAS Catalog of published genome-wide association studies, targeted arrays and summary statistics 2019. Nucleic Acids Res. 2019, 47, D1005-D1012. [CrossRef]

16. Cherlin, S.; Lewis, M.J.; Plant, D.; Nair, N.; Goldmann, K.; Tzanis, E.; Barnes, M.R.; McKeigue, P.; Barrett, J.H.; Pitzalis, C.; et al. Investigation of genetically regulated gene expression and response to treatment in rheumatoid arthritis highlights an association between IL18RAP expression and treatment response. Ann. Rheum. Dis. 2020, 79, 1446-1452. [CrossRef]

17. Portella, A.K.; Papantoni, A.; Paquet, C.; Moore, S.; Rosch, K.S.; Mostofsky, S.; Lee, R.S.; Smith, K.R.; Levitan, R.; Silveira, P.P.; et al. Predicted DRD4 prefrontal gene expression moderates snack intake and stress perception in response to the environment in adolescents. PLoS ONE 2020, 15, e0234601. [CrossRef]

18. Wheeler, H.E.; Ploch, S.; Barbeira, A.N.; Bonazzola, R.; Andaleon, A.; Fotuhi Siahpirani, A.; Saha, A.; Battle, A.; Roy, S.; Im, H.K. Imputed gene associations identify replicable trans-acting genes enriched in transcription pathways and complex traits. Genet. Epidemiol. 2019, 43, 596-608. [CrossRef] [PubMed]

19. Hu, Y.; Graff, M.; Haessler, J.; Buyske, S.; Bien, S.A.; Tao, R.; Highland, H.M.; Nishimura, K.K.; Zubair, N.; $\mathrm{Lu}, \mathrm{Y}$; et al. Minority-centric meta-analyses of blood lipid levels identify novel loci in the Population Architecture using Genomics and Epidemiology (PAGE) study. PLoS Genet. 2020, 16, e1008684. [CrossRef]

20. Fiorica, P.N.; Wheeler, H.E. Transcriptome association studies of neuropsychiatric traits in African Americans implicate PRMT7 in schizophrenia. PeerJ 2019, 7, e7778. [CrossRef] 
21. Bien, S.A.; Su, Y.R.; Conti, D.V.; Harrison, T.A.; Qu, C.; Guo, X.; Lu, Y.; Albanes, D.; Auer, P.L.; Banbury, B.L.; et al. Genetic variant predictors of gene expression provide new insight into risk of colorectal cancer. Hum. Genet. 2019, 138, 307-326. [CrossRef]

22. Xia, Z.; Su, Y.R.; Petersen, P.; Qi, L.; Kim, A.E.; Figueiredo, J.C.; Lin, Y.; Nan, H.; Sakoda, L.C.; Albanes, D.; et al. Functional informed genome-wide interaction analysis of body mass index, diabetes and colorectal cancer risk. Cancer Med. 2020, 9, 3563-3573. [CrossRef] [PubMed]

23. Ioannidis, N.M.; Wang, W.; Furlotte, N.A.; Hinds, D.A.; Me Research Team; Bustamante, C.D.; Jorgenson, E.; Asgari, M.M.; Whittemore, A.S. Gene expression imputation identifies candidate genes and susceptibility loci associated with cutaneous squamous cell carcinoma. Nat. Commun. 2018, 9, 4264. [CrossRef]

24. Pattee, J.; Zhan, X.; Xiao, G.; Pan, W. Integrating germline and somatic genetics to identify genes associated with lung cancer. Genet. Epidemiol. 2020, 44, 233-247. [CrossRef] [PubMed]

25. Li, B.; Verma, S.S.; Veturi, Y.C.; Verma, A.; Bradford, Y.; Haas, D.W.; Ritchie, M.D. Evaluation of PrediXcan for prioritizing GWAS associations and predicting gene expression. Pac. Symp. Biocomput. 2018, 23, 448-459.

26. Mikhaylova, A.V.; Thornton, T.A. Accuracy of Gene Expression Prediction From Genotype Data With PrediXcan Varies Across and Within Continental Populations. Front. Genet. 2019, 10, 261. [CrossRef] [PubMed]

27. Wheeler, H.E.; Shah, K.P.; Brenner, J.; Garcia, T.; Aquino-Michaels, K.; Consortium, G.T.; Cox, N.J.; Nicolae, D.L.; Im, H.K. Survey of the Heritability and Sparse Architecture of Gene Expression Traits across Human Tissues. PLoS Genet. 2016, 12, e1006423. [CrossRef]

28. Deutelmoser, H.; Scherer, T.; Brenner, H.; Waldenberger, M.; Study, I.; Suhre, K.; Kastenmüller, G.; Lorenzo Bermejo, J. Robust Huber-LASSO for improved prediction of protein, metabolite, and gene expression levels relying on individual genotype data. Brief. Bioinform. 2020, in press. [CrossRef]

29. Novembre, J.; Johnson, T.; Bryc, K.; Kutalik, Z.; Boyko, A.R.; Auton, A.; Indap, A.; King, K.S.; Bergmann, S.; Nelson, M.R.; et al. Genes mirror geography within Europe. Nature 2008, 456, 98-101. [CrossRef]

30. Chirshev, E.; Oberg, K.C.; Ioffe, Y.J.; Unternaehrer, J.J. Let-7 as biomarker, prognostic indicator, and therapy for precision medicine in cancer. Clin. Transl. Med. 2019, 8, 24. [CrossRef]

31. Liao, T.T.; Lin, C.C.; Jiang, J.K.; Yang, S.H.; Teng, H.W.; Yang, M.H. Harnessing stemness and PD-L1 expression by AT-rich interaction domain-containing protein 3B in colorectal cancer. Theranostics 2020, 10, 6095-6112. [CrossRef]

32. Kobayashi, K.; Era, T.; Takebe, A.; Jakt, L.M.; Nishikawa, S. ARID3B induces malignant transformation of mouse embryonic fibroblasts and is strongly associated with malignant neuroblastoma. Cancer Res. 2006, 66, 8331-8336. [CrossRef]

33. Joseph, S.; Deneke, V.E.; Cowden Dahl, K.D. ARID3B induces tumor necrosis factor alpha mediated apoptosis while a novel ARID3B splice form does not induce cell death. PLoS ONE 2012, 7, e42159. [CrossRef]

34. Akhavantabasi, S.; Sapmaz, A.; Tuna, S.; Erson-Bensan, A.E. miR-125b Targets ARID3B in Breast Cancer Cells. Cell Struct. Funct. 2012, 37, 27-38. [CrossRef] [PubMed]

35. Roy, L.; Samyesudhas, S.J.; Carrasco, M.; Li, J.; Joseph, S.; Dahl, R.; Cowden Dahl, K.D. ARID3B increases ovarian tumor burden and is associated with a cancer stem cell gene signature. Oncotarget 2014, 5, 8355. [CrossRef] [PubMed]

36. Lilla, C.; Verla-Tebit, E.; Risch, A.; Jäger, B.; Hoffmeister, M.; Brenner, H.; Chang-Claude, J. Effect of NAT1 and NAT2 Genetic Polymorphisms on Colorectal Cancer Risk Associated with Exposure to Tobacco Smoke and Meat Consumption. Cancer Epidemiol. Biomark. Prev. 2006, 15, 99-107. [CrossRef]

37. Brenner, H.; Chang-Claude, J.; Seiler, C.M.; Sturmer, T.; Hoffmeister, M. Does a negative screening colonoscopy ever need to be repeated? Gut 2006, 55, 1145-1150. [CrossRef] [PubMed]

38. Peters, U.; Jiao, S.; Schumacher, F.R.; Hutter, C.M.; Aragaki, A.K.; Baron, J.A.; Berndt, S.I.; Bezieau, S.; Brenner, H.; Butterbach, K.; et al. Identification of Genetic Susceptibility Loci for Colorectal Tumors in a Genome-Wide Meta-analysis. Gastroenterology 2013, 144, 799-807.e24. [CrossRef]

39. Ulrich, C.M.; Gigic, B.; Bohm, J.; Ose, J.; Viskochil, R.; Schneider, M.; Colditz, G.A.; Figueiredo, J.C.; Grady, W.M.; Li, C.I.; et al. The ColoCare Study: A Paradigm of Transdisciplinary Science in Colorectal Cancer Outcomes. Cancer Epidemiol. Prev. Biomark. 2019, 28, 591-601. [CrossRef] 
40. Haffa, M.; Holowatyj, A.N.; Kratz, M.; Toth, R.; Benner, A.; Gigic, B.; Habermann, N.; Schrotz-King, P.; Bohm, J.; Brenner, H.; et al. Transcriptome Profiling of Adipose Tissue Reveals Depot-Specific Metabolic Alterations Among Patients with Colorectal Cancer. J. Clin. Endocrinol. Metab. 2019, 104, 5225-5237. [CrossRef]

41. Sudlow, C.; Gallacher, J.; Allen, N.; Beral, V.; Burton, P.; Danesh, J.; Downey, P.; Elliott, P.; Green, J.; Landray, M.; et al. UK Biobank: An Open Access Resource for Identifying the Causes of a Wide Range of Complex Diseases of Middle and Old Age. PLoS Med. 2015, 12, e1001779. [CrossRef]

42. Bycroft, C.; Freeman, C.; Petkova, D.; Band, G.; Elliott, L.T.; Sharp, K.; Motyer, A.; Vukcevic, D.; Delaneau, O.; O'Connell, J.; et al. The UK Biobank resource with deep phenotyping and genomic data. Nature 2018, 562, 203-209. [CrossRef] [PubMed]

Publisher's Note: MDPI stays neutral with regard to jurisdictional claims in published maps and institutional affiliations.

(C) 2020 by the authors. Licensee MDPI, Basel, Switzerland. This article is an open access article distributed under the terms and conditions of the Creative Commons Attribution (CC BY) license (http://creativecommons.org/licenses/by/4.0/). 